Research Paper

\title{
The Impact of a Nationwide Antibiotic Restriction Program on Antibiotic Usage and Resistance against Nosocomial Pathogens in Turkey
}

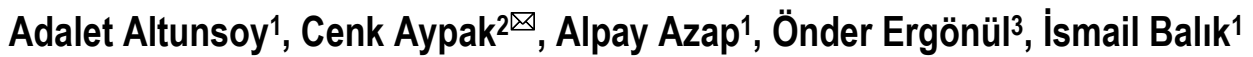 \\ 1. Department of Clinical Microbiology and Infectious Disease, Ankara University, School of Medicine, Ibni Sina Hospital \\ 06100, Ankara, Turkey \\ 2. Department of Family Medicine, Ankara University, School of Medicine, Ibni Sina Hospital 06100, Ankara, Turkey \\ 3. Department of Clinical Microbiology and Infectious Disease, Marmara University, School of Medicine, 34662, Istanbul, \\ Turkey
}

Corresponding author: cenkaypak@gevasdh.gov.tr; Tel: +90 505 6452780; Fax: +90 3123240328

(๑) Ivyspring International Publisher. This is an open-access article distributed under the terms of the Creative Commons License (http://creativecommons.org/ licenses/by-nc-nd/3.0/). Reproduction is permitted for personal, noncommercial use, provided that the article is in whole, unmodified, and properly cited.

Received: 2011.02.22; Accepted: 2011.05.16; Published: 2011.05.24

\begin{abstract}
Purpose: Antimicrobial resistance among microorganisms is a global concern. In 2003, a nationwide antibiotic restriction program (NARP) was released in Turkey. In this study we evaluated the effect of NARP on antibiotic consumption, antimicrobial resistance, and cost.

Materials and Methods: The data obtained from all of the four university hospitals, and one referral tertiary-care educational state hospital in Ankara. Antimicrobial resistance profiles of 14,233 selected microorganisms all grown in blood cultures and antibiotic consumption from 2001 to 2005 were analyzed retrospectively.

Results: A negative correlation was observed between the ceftriaxone consumption and the prevalence of ceftriaxone resistant E.coli and Klebsiella spp. (rho:-0.395, p:0.332 and rho:-0.627, $p: 0.037$, respectively). The decreased usage of carbapenems was correlated with decreased carbapenems-resistant Pseudomonas spp. and Acinetobacter spp (rho:0.155, $p: 0.712$ and rho:0.180, $p: 0.668$, respectively for imipenem). Methicillin resistance rates of S.aureus were decreased from $44 \%$ to $41 \%$. After two years of NARP $5,389,155.82$ USD saving occurred.

Conclusion: NARP is effective in lowering the costs and antibiotic resistance.
\end{abstract}

Key words: Antibiotic consumption, antimicrobial resistance surveillance, restriction policy.

\section{Introduction}

It is obvious that antibiotics had saved many lives since they were first introduced to medical practice. However, when antibiotics are used the emergence of drug resistant microorganisms is inevitable. The emergence of resistant microorganisms becomes faster when antibiotic use is inappropriate [1]. As well as emergence of resistant microorganisms, increased mortality and morbidity, adverse drug reactions and excessive strain on already limited healthcare budgets are the results of inappropriate antibiotic consumption [2-4]. These findings provide compelling evidence of the need for more rational use of antimicrobial agents in all over the world [5-9]. In order to slowdown the development and dissemination of resistant bacteria, restrictions on antibiotic prescribing are becoming more widespread [10].

In February 2003, Turkish Ministry of Health released a nationwide regulation for antibiotic re- 
striction. According to nationwide antibiotic restriction program (NARP), carbapenems, glycopeptids, piperacillin/tazobactam, ticarcillin/clavulanate were considered as restricted antibiotics that could be used only with the approval of an infectious disease specialist (IDS). Parenteral quinolones, 3(rd) and 4(th) generation cephalosporins, netilmisin, amikacin could still be prescribed by all specialists just for the first 72 $\mathrm{h}$ of treatment but further utilization required IDS approval.

In this multicenter study we aimed to assess the impact of the antibiotic restriction policy on the antibiotic use, financial cost and resistance patterns of leading nosocomial pathogens.

\section{Materials and Methods}

Hospital setting and antibiotic policy: NARP was initiated in Turkey in February 2003 by a central regulation of Ministry of Health and was announced nation-wide via official newspaper of the state [11]. This is a quasi-experimental study performed in four year period, which included two years before and after of the initiation of NARP in 2003. The study included the data obtained from all of the four university hospitals, and one referral tertiary-care educational state hospital in Ankara. These hospitals have a total of 6668 beds.

Microbiologic studies: Microbiology laboratory results of hospitals were evaluated retrospectively. Significant nosocomial pathogens, namely Pseudomonas spp., Escherichia coli, Klebsiella spp., Acinetobacter spp., Staphylococcus aureus obtained from at least one set of blood cultures of the inpatients were included. More than one set of the same isolates from the same patient were counted as one microorganism. All laboratories were using automatic blood culture systems (Bac-Tec ${ }^{\circledR}$ Becton-Dickinson, BacT-ALERT ${ }^{\circledR}$ BioMerieux) and performing antimicrobial resistance testing by Kirby Bauer disc diffusion method according to the recommendations of Clinical Laboratory Standart Institute (CLSI) [12]. Resistance patterns of ciprofloxacin, 3(rd) and 4(th) generation cephalosporins, (ceftazidime, ceftriaxone, cefepime), piperacillin-tazobactam, carbapenems (imipenem, meropenem), aminoglycosides (amikacin, gentamicin) against aforementioned pathogens were analysed. Bacterial idenfications were performed by conventional methods and automatic systems (API 20E ${ }^{\circledR}$ strips BioMerieux, BBL Crystal ${ }^{\circledR}$ Becton-Dickinson).

Antibiotic expenditure and cost: Aggregate amount of antibiotic consumption as total weight (gram) and number of boxes were calculated from two databases, 1) Hospital pharmacy computer databases, and 2) International Medication System (IMS). Because Turkey is an inflation country we have escalated all antibiotic prices. The cost of antibiotics was calculated as US dollars (USD).

Statistical Analysis: Rates in every 6 months periods of the study period were analyzed by comparison of proportions with the chi-square test. Correlations between antibiotic resistance and consumption calculated by two-tailed Spearman's coefficient $(r)$ for non-parametric correlations. A $P$ value of less than 0.05 was regarded as significant. Software package STATA 9.0 (USA) was used for the analysis.

\section{Results}

In total, 14,233 microorganisms were enrolled in the study from 2001 to 2005. Of which 5371 were E.coli, 1323 Klebsiella spp., 1101 Acinetobacter spp., 1637 Pseudomonas spp., 4801 S.aureus. Data on bacterial resistance are summarized in table 1.

Changes in the consumption of given antimicrobials for two years before and after the initiation of NARP can be seen in table 2 .

A negative correlation was observed between the ceftriaxone consumption and the prevalence of ceftriaxone resistant E.coli and Klebsiella spp. (rho:-0.395, $p=0.332$ and rho:-0.627, $p=0.037$, respectively).

Inspite of increased consumption of piperacillin-tazobactam after the NARP, the resistance rates of E.coli and Klebsiella spp. against piperacillin-tazobactam did not increase significantly (rho:0.626, $p=0.096$ and rho:0.357, $p=0.385$, respectively).

The decreased use of carbapenems was correlated with decreased rate of carbapenem-resistant Pseudomonas spp. and Acinetobacter spp (Spearman rho:0.155, $p=0.712$ and Spearman rho:0.180, $p=0.668$, respectively).

Ceftazidim utilization and resistance rate of Pseudomonas spp. to this agent both had downward tendency after NARP. Also methicillin resistance rates of S.aureus were decreased from $44 \%$ to $41 \%$ during the study period. However, this relationship was not statically significant $(p=0,866)$.

The cost of antibiotic utilization before and after NARP for selected drugs is shown in Table 3. It was found out totally 5,389,155.82 USD saved in the budget for two years period. 
Table 1. Impact of NARP* on bacterial resistance rates for the selected antibiotics

\begin{tabular}{llllc}
\hline & Resistance rate (\%) & & \\
\hline & Before NARP & After NARP & Difference & $p$ Value \\
E.coli / ceftriaxone & 2001 and 2002 & 2003 and 2004 & +12.8 & NS \\
E.coli / PIP-TAZO ** & 22 & 34.8 & +7.5 & NS \\
Klebsiella/ceftriaxone & 16.8 & 24.3 & +10 & NS \\
Klebsiella/ PIP-TAZO ** & 29.3 & 39.3 & +8.3 & NS \\
Acinetobacter/imipenem & 25.5 & 33.8 & -6.3 & NS \\
Pseudomonas/ceftazidim & 51.3 & 45 & -5.7 & NS \\
Staph. Aureus/methicillin & 48.5 & 42.8 & -3.0 & NS \\
\hline
\end{tabular}

*nationwide antibiotic restriction program, ${ }^{* *}$ piperacillin-tazobactam

NS:not significant, $\mathrm{p}>0.05$.

Table 2: Comparison of antibiotic consumption two years before and after the initiation of NARP*

\begin{tabular}{llll}
\hline Restricted Antibiotics & Antibiotic consumption (grams) & \multicolumn{2}{c}{ \% difference } \\
\cline { 2 - 4 } & $2001+2002$ & $2003+2004$ & -24.8 \\
\hline Meropenem & 113362 & 85236 & -9.1 \\
Imipenem & 50532 & 45935.2 & -36.5 \\
Ceftazidim & 60074 & 38129 & -36.8 \\
Ceftriaxone & 300955 & 190281 & +54.1 \\
PIP-TAZO* & 270594 & 417114 & +21.1 \\
Cefepime & 100588 & 121799 & -17.8 \\
Vancomycin & 113362 & 85236 & -1.4 \\
Teicoplanin & 50532 & 45935.2 & $\mathbf{- 1 1 . 3}$ \\
Total & 60074 & 38129 & \\
\hline
\end{tabular}

*nationwide antibiotic restriction program, ** piperacillin-tazobactam

Table 3. Comparison of cost of antibiotics

\begin{tabular}{lllc}
\hline Restricted Antibiotics & Cost (US \$) & $2003+2004$ & \% difference \\
\cline { 2 - 4 } & $2001+2002$ & $7,156,244.09$ & -24.8 \\
\hline Meropenem & $9,517,646.80$ & $3,389,099.06$ & -9.1 \\
Imipenem & $3,728,250.96$ & $989,676.32$ & -36.5 \\
Ceftazidim & $1,559,280.74$ & $5,024,179.52$ & -36.8 \\
Ceftriaxone & $7,946,415.82$ & $3,561,111.98$ & +54.1 \\
PIP-TAZO* & $2,310,030.91$ & $2,322,463.33$ & +21.1 \\
Cefepime & $1,918,011.98$ & $2,797,636.80$ & -17.8 \\
Vancomycin & $3,403,176.00$ & $17,081,283.38$ & -1.4 \\
Teicoplanin & $17,328,037.09$ & $\mathbf{4 2 , 3 2 1 , 6 9 4 . 4 8}$ & $\mathbf{- 1 1 . 3}$ \\
Total & $\mathbf{4 7 , 7 1 0 , 8 5 0 . 3 0}$ & & \\
\hline
\end{tabular}

*piperacillin-tazobactam 


\section{Discussion}

Antibiotics are among the most frequently prescribed drugs. A close association exists between resistance rate and the amount of antimicrobial agents used [1]. This indicates a serious need to control antibiotic consumption. Optimization of antibiotic usage not only prevents increase in resistance but also cuts down the healthcare costs.

Several strategies for regulating antimicrobial prescribing have been proposed, such as health care provider educational programmes, development of prescribing guidelines, monitoring resistance patterns, feedback activities, introduction of order forms, formulary replacement or institutional restrictions, and limitation of contacts between physicians and pharmaceutical representatives [13-16]. It has been reported that the requirement for approval from an IDS is the most effective control method [17, 18]. The studies on antibiotic restriction policies are generally about financial concerns and antibiotic utilization but the bacterial resistance are not usually co analysed [19]. We conducted this comprehensive multi centric study to evaluate the effect of a nationwide restriction programme on both antibiotic consumption and antimicrobial resistance rates.

Few hospitals had a restriction policy before 2003 in Turkey. Five tertiary-care educational hospitals from which we collected data for this study had already applied a local antibiotic restriction policy and all five centres had founded infection control committees many years before the initiation of the NARP. Even in these selected centres already applying local antibiotic restriction policies the utilisation of many of the restricted antibiotics was decreased and the trend of resistance rates became downwards after implementation of NARP. The amount of money saved increased further. After two years of NARP $5,389,155.82$ USD saving occurred in the selected drugs. The restriction policy has resulted in clear and immediate saving. The long term influence on medical budget may be stronger than the beginning. The financial impact of antimicrobial restriction program has been shown both in developed and developing countries [6, 17, 20-23].

The resistance rates of given microorganisms for all of the antibiotics evaluated were not increased significantly. For instance in spite of increased consumption of piperacilin-tazobactam (TZP) after NARP resistance rates did not increase significantly. This finding for TZP is in accordance with the literature [24]. This finding has revealed that restricted antimicrobials has been started to be utilized more rationale after the initiation of NARP. Also carbapenem resistance rates of Pseudomonas spp and Acinetobacter spp decreased correlating with decreased consumption of carbapenems after NARP (Spearman rho:0.155, $p: 0.712$ and Spearman rho:0.180, $p: 0.668$, respectively for imipenem). Falagas et al. reported decreased resistance rates of Pseudomonas aeruginosa but not of Acinetobacter baumannii and E. coli isolates by restriction policy [22]. Regal et al. have found imipenem resistance of Pseudomonas aeruginosa declined fom $20.5 \%$ to $12.3 \%$ with an $18 \%$ reduction in use [25]. A negative correlation was observed between the ceftriaxone consumption and the prevalence of ceftriaxone resistant E.coli and Klebsiella spp. (Spearman rho:-0.395, $p: 0.332$ and Spearman rho:-0.627, $p: 0.037$, respectively). This finding may partially be explained by a shift in antibiotic consumption toward unrestricted drugs such as second and third generation oral cephalosporines. High cephalosporine use is a well-known risk factor for emergence of ESBL producing Enterobacteriacea [26]. It was shown that inappropriate antibiotic use was significantly higher among unrestricted antibiotics than restricted ones in a study comparing antibiotic utilisation before and after NARP in a single centre from Turkey revealed $125.3 \%$ increase in the use of $2^{\text {nd }}$ and $3^{\text {rd }}$ generation oral cephalosporins [27]. Furthermore this finding was confirmed by other studies from different parts of world [21,22]. The shift toward unrestricted antibiotics changes the antimicrobial resistance patterns of certain pathogens. Since parenteral forms of ciprofloxacin and levofloxacin were not restricted for the first three days of therapy by NARP, the consumption of these quinolones was unsurprisingly high. Besides that the use of oral quinolones for maintenance may contribute to the significant increase in prevalence of quinolone resistant E.coli strains [28, 29].

There are several limitations of our study. First, we were not able to investigate whether restrictive use of antibiotics in these five tertiary-care settings was associated with a change in frequency of deaths or nursing expenses. Second, we investigated only the restricted antibiotics because of this we do not know the consumption rate of the antibiotics which can be prescribed by all physicians. Third, the study period after NARP may not be long enough to see the changes in antimicrobial resistance. It should be kept in mind that there is a time lag between antibiotic use and possible changes in antibiotic resistance. Austin et al. showed that the time scale for emergence of resistance under constant selective pressure is much shorter than decay time after cessation or decline in the level of drug use [30]. Enne et al. showed that a huge decrease in sulphonamide prescribing in the UK did not have an effect on the prevalence of resistance 
to this drug in E.coli within a useful time [31]. Although this study comprises two years after the initiation of restriction policy there is still a need for continuous surveillance studies to observe the full impact of the NARP. Fourth, we calculated antibiotic consumption in grams instead of using "daily defined dose" (DDD) to evaluate the consumption because of some concerns. The DDD is a technical unit which is the assumed average maintenance dose per day for the drug's main indication in adults and is assigned by the WHO collaborating centre [32]. Expression of data for antibiotic consumption in DDDs might not adequately address differences in dosage and for specific classes of antibiotics between centres. Also, DDDs do not take into account different doses for children. Hence the use of DDDs for adults to express children's consumption might lead to under presentation of this segment of users in total. All five hospitals in this study have their own paediatric disease wards with 839 beds totally.

In conclusion, although our study has aforementioned limitations and the antibiotic restriction is a controversial issue from many points of view (ethical, pharmaceutical, patient benefit etc.), this is the first multi centric study from Turkey which evaluates the effect of NARP on both antibiotic consumption and antimicrobial resistance rates and indicates that NARP in Turkey was effective in lowering the costs and antibiotic resistance.

\section{Acknowledgements}

The authors thank to Özay Akan, Yeşim Çetinkaya Şardan, Gülşen Hasçelik, Deniz Gür, Furdevs Aktaş, Dilek Arman, Nedim Sultan, Bülent Beşirbellioğlu and Esra Karakoç for their kindness in data collection.

\section{Conflict of Interest}

The authors have declared that no conflict of interest exists.

\section{References}

1. Fishman ON. Antimicrobial Stewardship. Am J Med. 2006; 119 (6A): 53-61.

2. Polk ER, Fishman ON. Antimicrobial Management: Cost and Resistance. In: Mandell GL, Bennett JE, Dolin R, eds. Principles and Practice of Infectious Diseases, 6th ed. Philadelphia: Churchill Livingstone Inc; 2005: 611-619.

3. McGowan JEJr. Economic Impact of Antimicrobial Resistance. Emerg Infect Dis. 2001; 7:286-292.

4. Polk R. Optimal Use of Modern Antibiotics: Emerging Trends. Clin Infect Dis. 1999; 29: 264-274.

5. Bassetti M, Di Biagio A, Rebesco B, Amalfitano ME, Topal J, Bassetti D. The effect of formulary restriction in the use of antibiotics in Italian hospital. Eur J Clin Pharmacol 2001; 57:529-534.

6. Ruttimann S, Keck B, Hartmeier C, Maetzel A, Bucher HC. Long term antibiotic cost savings from a comprehensive inter- vention program in a medical department of a university affliated teaching hospital. Clin Infect Dis. 2004; 38:348-356.

7. Bantar C, Sartori B, Vesco E, Heft C, Saúl M, Salamone F, Oliva ME. A hospital wide intervention program to optimize the quality of antibiotic use: Impact on prescribing practice, antibiotic consumption, cost savings, and bacterial resistance. Clin Infect Dis. 2003; 37:180-186.

8. Vlahovic-Palcevski V, Morovic M, Palcevski G. Antibiotic utilization at the university hospital after introducing an antibiotic policy. Eur J Clin Pharmacol. 2000; 56:97-101.

9. Saizy-Callaert S, Causse R, Furhman C, Le Paih MF, Thébault A, Chouaïd C. Impact of a multidisciplinary approach to the control of antibiotic prescription in a general hospital. J Hosp Infect. 2003; 53:177-182.

10. Garau J. Impact of antibiotic restrictions: The ethical perspective. Clin Microbiol Infect. 2006; 12(suppl 5):16-24.

11. No authors listed. 1 February 2003, No: 25011. Turkish official gazette. 2003.

12. National Committee for Clinical Laboratory Standards. Performance Standards for Antimicrobial Susceptibility Testing. Wayne, PA, USA: 13th Informational Supplement M100-S13 NCCLS; 2003.

13. Rubin MA, Samore MH. Antimicrobial use and resistance. Curr Infect Dis Rep. 2002; 4:491-497.

14. Gyssens IC, Meer JWM. Quality of antimicrobial drug prescription in hospital. Clin Microbiol Infec. 2001; 7:12-15.

15. Couper MR. Strategies for the rational use of antimicrobials. Clin Infect Dis. 1997; 24:54-56.

16. le Grand A, Hogerzeil HV, Haaijer-Ruskamp FM. Intervention research in rational use of drugs: a review. Health Policy Plan. 1999; 14:89-102.

17. Hirschman SZ, Meyers BR, Bradbury K, Mehl B, Gendelman S, Kimelblatt B. Use of antimicrobial agents in a university teaching hospital. Evolution of comprehensive control program. Arch Intern Med. 1988; 148:2001-2007.

18. McGowan JEJr. Minimizing antimicrobial resistance: The key role of the infectious diseases physician. Clin Infect Dis. 2004; 38:939-942.

19. Wilton P, Smith R, Coast J, Millar M. Strategies to contain the emergence of antimicrobial resistance: a systematic review of effectiveness and cost effectiveness. J Health Serv Res Policy. 2002; 7:111-116.

20. John JF, Fishman ON. Programmatic role of the infectious diseases physician in controlling antimicrobial costs in the hospital. Clin Infect Dis. 1997; 24:471-485.

21. Bassetti M, Biagio AD, Rebesco B, Cenderello G, Amalfitano ME, Bassetti D. Impact of an antimicrobial formulary and restriction policy in the largest hospital in Italy. Int J Antimicrob Agents. 2000; 16:295-299.

22. Falagas ME, Bliziotis IA, Michalopoulos A, Sermaides G, Papaioannou VE, Nikita D, Choulis N. Effect of a policy for restriction of selected classes of antibiotics on antimicrobial drug cost and resistance. J Chemother. 2007; 19(2):178-184.

23. Siddiqui S, Hussein K, Manasia R, Samad A, Salahuddin N, Zafar A, Hoda MQ. Impact of antibiotic restriction on broad spectrum antibiotic usage in the ICU of a developing country. J Pak Med Assoc. 2007; 57(10):484-487.

24. Petrikkos G, Markogiannakis A, Papaparaskevas J, Daikos GL, Stefanakos G, Zissis NP, Avlamis A. Differences in the changes in resistance patterns to third- and fourth-generation cephalosporins and piperacillin/tazobactam among Klebsiella pneumoniae and Escherichia coli clinical isolates following a restriction policy in a Greek tertiary care hospital. Int J Antimicrob Agents. 2007; 29(1):34-38.

25. Regal RE, DePestel DD, VandenBussche HL. The effect of an antimicrobial restriction program on Pseudomonas aeruginosa 
resistance to beta-lactams in a large teaching hospital. Pharmacotherapy. 2003; 23(5):618-624.

26. Bantar C, Vesco E, Heft C, et al. Replacement of broad-spectrum cephalosporins by piperacillin-tazobactam: Impact on sustained high rates of bacterial resistance. Antimicrob Agents Chemother. 2004; 48(2):392-395.

27. Ozkurt Z, Erol S, Kadanali A, Ertek M, Ozden K, Tasyaran MA. Changes in antibiotic use, cost and consumption after an antibiotic restriction policy applied by infectious disease specialists. Jpn J Infect Dis. 2005; 58(6):338-343.

28. Yagci D, Yoruk F, Azap A, Memikoglu KO. Prevalence and risk factors for selection of quinolone-resistant Escherichia coli strains in fecal flora of patients receiving quinolone therapy. Antimicrob Agents Chemother. 2009; 53(3):1287-1289.

29. Aypak C, Altunsoy A, Düzgün N. Empiric antibiotic therapy in acute uncomplicated urinary tract infections and fluoroquinolone resistance: a prospective observational study. Ann Clin Microbiol Antimicrob. 2009; 24(8):27.

30. Austin DJ, Kristinsson KG, Anderson RM. The relationship between the volume of antimicrobial consumption in human communities and the frequency of resistance. Proc Natl Acad Sci USA. 1999; 96:1152-1156

31. Enne VI, Livermore DM, Stephens P, Hall LM. Persistence of sulphonamide resistance in Escherichia coli in the UK despite national prescribing restriction. Lancet. 2001; 357(9265): 1325-1328.

32. Knox KL, Holmes AH. Regulation of antimicrobial prescribing practices: a strategy for controlling nosocomial antimicrobial resistance. Int J Infect Dis. 2002; 6(1):8-13. 